\title{
Metamodels for Information System Modeling in Production Environments
}

\author{
G.S. Mannarino*, G.P. Henning+ and H.P. Leone*. \\ * Instituto de Desarrollo y Diseño (INGAR), Fundación ARCIEN - \\ CONICET, GIPSI-FRSF-Univ. Tecnológica Nacional \\ Avellaneda 3657, 3000 Santa Fe, Argentina, Tel.: 5442 553439, \\ Fax: 5442 553439, E-mail: hleone@arcride.edu.ar \\ + Instituto de Desarrollo Tecnológico para la Industria Química \\ (INTEC), CONICET - UNL
}

Güemes 3450, 3000 Santa Fe, Argentina, Tel.: 5442 559175, Fax: 5442 550944, E-mail: ghenning@intec.unl.edu.ar

\begin{abstract}
Production environment models can assist the goal of enterprise integration by helping to represent, and later, analyze and evaluate the structure of the enterprise activities and the interactions among them. This paper presents advances in the development of an open model architecture consisting of three layers: (i) Metamodel layer, (ii) Model layer and (iii) Universe of Discourse layer. The contribution focuses on the introduction of task and domain metamodels to be used as templates in the construction of a production environment model library. In order to develop such models the Object Technology, enriched with the concepts of version, perspective and evolution was adopted.
\end{abstract}

\section{Keywords}

Enterprise Integration. Enterprise Modeling. Object Oriented Analysis. Computer Integrated Manufacturing.

\section{INTRODUCTION}

Modeling is an invaluable approach to addressing the Enterprise Integration (EI) Problem. Several important modeling methodologies, from different application domains, have been 
proposed in this field (Vernadat, 1992; Berio, 1995). The first International Conference on Enterprise Integration Modeling Technology (ICEIMT) roughly identified the three following types of approaches to the problem of syntactic and semantic model integration: Master, Unified and Federated Models (Petrie, 1992). This paper presents advances in the development of a set of metamodels that belongs to the first group. The proposed metamodels allow the description of a variety of production facilities, acting as a foundation to the analysis and design of an integrated information system.

This work focuses on the introduction of a set of task and domain metamodels, based on the Object Technology (Stefik, 1985; Kiczales et al., 1991; Booch, 1994; Jacobson, 1994), to be used as templates in the construction of models of production environments. The primary reason to develop such models is to supply a mechanism for understanding, analyzing and evaluating the production enterprise.

The aim of task metamodels is to describe an organization in terms of (i) the activities that are to be performed so as to achieve the organization purposes such as Production Planning, Product and Process Engineering, Marketing, Distribution, Business Management, etc., (ii) the physical and information resources that are utilized by each task performed in the organization and (iii) the role that each resource plays in relation to a task (whether it can be consumed, modified, used exclusively by the task, etc.)

Domain models focus on the identification of the entities that represent an organization, their relationships, and properties. For example, they will classify batch plants in terms of their operating strategies (i.e., multiproduct, multipurpose and multiplant), an equipment item in terms of its functionality, a production campaign in terms of its starting and finishing times, the product it manufactures, the equipment unit it uses, etc.

\section{ANALYSIS OF THE PROPOSED METAMODELS}

Integrated production facilities should properly administrate a huge amount of information having a very complex structure and a variety of formats, such as text, graphics, constraints, engineering procedures, etc. (Vernadat, 1988; Meyer, 1990). Therefore, the requirement exists for an appropriate representation mechanism able to express all sorts of enterprise knowledge. The Object Oriented Paradigm, enriched with the concepts of version, perspective and evolution (Mattsson, 1988; Stephanopoulos et al., 1990; Ziegler, 1984; Li and McLeod, 1994; Stefik, 1985), was adopted to develop the proposed representation.

The task and domain object metamodels presented in this paper are first introduced by means of a pictorial notation that is a modified version of the one proposed by Martin and Odell (1992). However, models are later expressed in terms of a formal grammar that defines the modeling language primitives (i.e., objects and relationships linking them) as well as cardinality constraints that apply on relationships. Having such a grammar can help in the verification of the models obtained for a specific production environment when applying the metamodels that are proposed in this contribution.

The suggested model architecture is depicted in Figure 1. This figure, showing three different layers of models, indicates that each layer depends on the primitives provided by the previous layer. The bottom level represents the Metamodel layer. At this layer, generic constructions that pertain to production environments and are independent of any application, are introduced. Classes defined at this layer encapsulate the fundamental model concepts and, 
more importantly, the production environment underlying semantics, e.g., the notion of function, task, resource, etc.

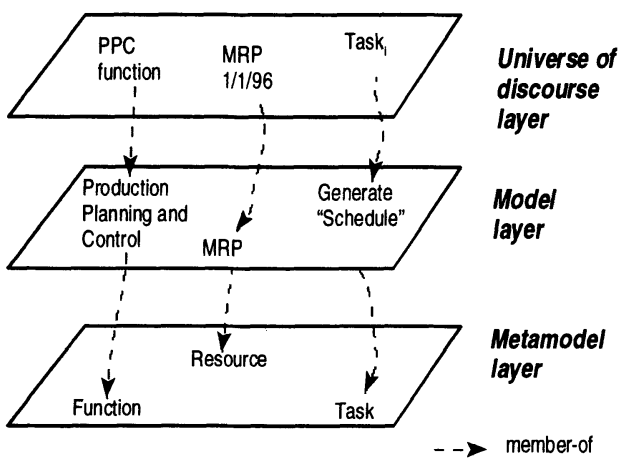

Figure 1 Three level architecture that links the Universe of Discourse, the Model layer and the Metamodel layer.

In the next level, associated with the Model layer, the characteristics of the application domain are abstracted. This layer reflects, through different models, the operating procedures, tasks and resources associated to industrial enterprises. The objective is to develop an open model library, that could be specialized to reflect the peculiarities of different types of production facilities. This library contains, among others, the following models: organizational structure, functional structure, tasks involved in the different enterprise activities, etc. When these models are specialized, new models belonging to the same level are generated. However, this layer is also "open" to the generation of new models that could be developed from the instantiation of metalevel primitives. Finally, the top level, called Universe of discourse layer, contains the real world and conceptual entities (instances of the objects defined at the previous level) that constitute the actual models of specific production facilities.

\subsection{Metamodel Primitives}

The primitive objects that belong to the metamodel level are depicted in Figure 2 (See Figure 3 for notation conventions). They are: (1) Basic class, (2) Relationship and (3) Perspective. Basic class represents objects that allow the description of the application domain. A Basic class is characterized by a set of attributes and relationships that link it with other domain objects. At this level object behavior is not considered. Basic classes are, among others, Function, Task, Objective, Goal, Resource and Organizational Unit. Some considerations regarding the contents of Figure 2 have to be made since it expresses the cartesian product of Basic Class and Relationship. Without introducing any additional constraints this fact would allow, for instance, a Resource to be composed-of a Task. Therefore, some constraints have to be defined. For the sake of simplicity only two of them are shown at the right bottom of the model. 
When modeling an organization, the developed models should reflect what happens in it. A production environment is far from being a static structure. For example, during product or process design activities, sequences of prototypes are generated till reaching final versions that meet the requirements imposed by different enterprise departments such as Production, Engineering, Finance, etc. Similarly, product specifications can be modified along product life cycle due to changes in customer requirements, quality improvements, recipe modifications, etc. In order to capture the previous ideas, an object version is defined as an alternative of a particular class. Two class objects are related by a "gives-rise-to" link to express the fact that both constitute different versions of the same entity.

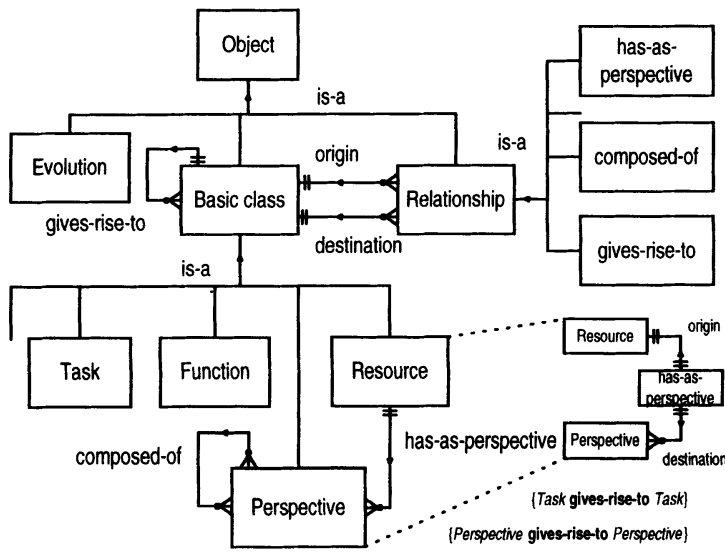

Figure 2 Metamodel layer primitives.

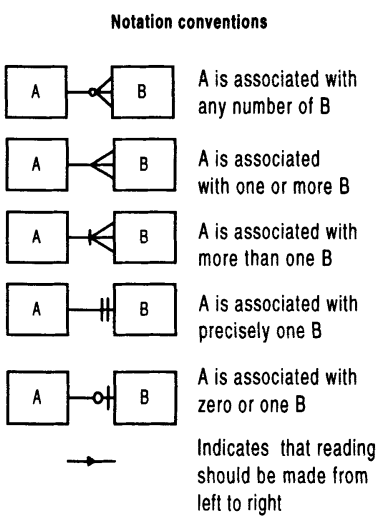

Figure 3 Notation conventions.

A Relationship object links two class objects. It is identified by a label and has a minimum $(0,1)$ and maximum ( 1 or greater than 1$)$ origin and destination cardinality. The name of a relationship describes the type of role that the destination object plays in relation to the origin one.

A role is defined as the ability or capability that an object has when it participates in a specific relationship with another object. In order to represent the different roles of an object the Perspective construction is used. As seen in Figure 2, Resource is defined as a composite object. Each Resource component has a perspective that represents information pertaining to a given role of the resource. The link that relates an object with its perspective is called "hasas-perspective", and its inverse relationship is named "is-a-perspective-of". A Resource may have zero or more perspectives, but a perspective is associated with only one Resource. Thus, the perspective idea allows resources to be seen in different ways according to the tasks they are involved into. For example, as seen in Figure 4(a), information concerning a product manufactured by a given company could be organized according to the following perspectives: (1) Product Market Data, that encapsulates information relative to price, sale conditions, type of package, etc.; (2) Product Inventory Data, that represents information regarding to storage conditions, actual level of stock, stock security level, etc.; (3) Product Planning Data, that maintains information on the allowed and average scrap levels, standard processing times, etc.; (4) Product Engineering Data, that summarizes data about product 
formula, associated recipe, etc.; (5) Finished product; (6) Intermediate product and (7) Raw material. These last three perspectives take into account the production plant where the product is utilized/produced and the processes it is involved into. Figure 4(b) shows that raw materials, intermediate and finished products are organized in a composition hierarchy to represent the structure of a product. However, some constraints should be added to this metamodel to make it meaningful. For instance, an intermediate product is such if it is composed of either raw materials or other intermediate products.

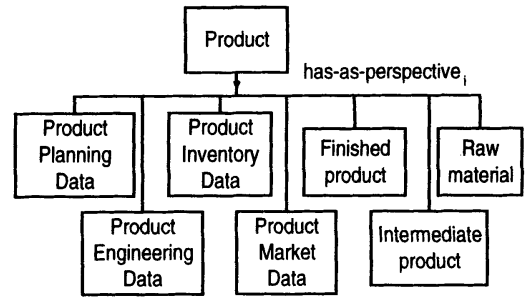

Figure 4 Product Domain Models.

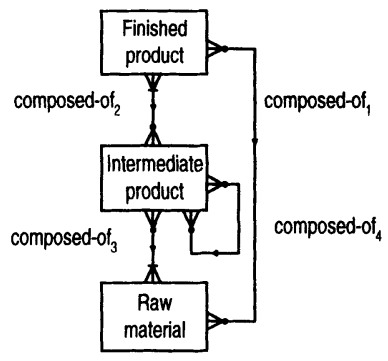

(b) Composition hierarchy representing a product structure.

The structure and behavior (if it were considered) of the objects identified in the different metamodels are given by a metaclass called Metaclass. According to Kiczales et al. (1991) different Metaclass specializations allow the definition of the basic object-oriented paradigm. Since Metaclass is not a concept that pertains to the domain being studied, it is not shown in the previous models. Thus, this paper focuses on the use of metamodels as templates employed to represent production environments, and it does not address the way metamodels are constructed as instances of higher level classes.

\subsection{Enterprise Metamodel}

One of the main metamodels presented in this paper, referred to as a "Corporate or Enterprise" metamodel, relates organizational units with functions (See Figure 5). In order to identify the system being studied an Organizational unit object is employed. This object represents the organization being analyzed in relation to other organizations, as well as its decomposition in different subsystems (division, department, plant, storage area, etc.). In this way, interactions with customers, raw material suppliers and service providers receive the same characterization as interactions between internal departments. Thus, this metamodel supports integration across the boundaries of the enterprise in the same way as it does internally. The proposed metamodel assumes that one or more responsibility lines are present. This is expressed through the "composed-of" relation shown in Figure 5; it links two Organizational unit objects and has a one or greater than one cardinality.

According to this metamodel, any Organization unit has two perspectives: client and server. Therefore, the whole enterprise can be abstractly seen as a service organization. For instance, 
the Planning Department supplies services to (i) the Production Department, in the form of production plans, (ii) the Purchase Department, in the form of material requirements, etc. On the other hand, it acts as a client when it receives information about (i) sale forecasts and firm customer orders from the Sales Department, (ii) product recipes and bills of material from the Product Engineering Department and (iii) processing capacities and equipment availability from the Production Area. Services provided by an Organizational unit are carried out as Functions performed by it.

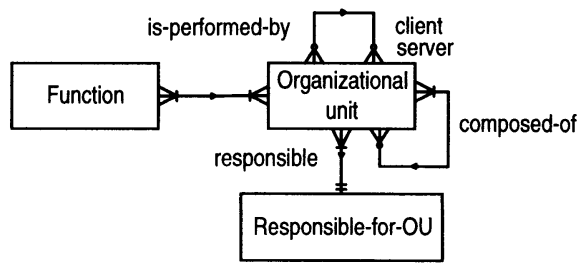

Figure 5 Enterprise metamodel.

Functions represent activities that are continuously carried out in a corporation such as Planning, Production, Research, Accounting, etc. The identification of Functions should represent fundamental concern for how the corporation operates. Though this metamodel links Functions with Organizational units by resorting to "is-performed-by" relationships, these links may change quite often since some corporations reorganize their structure frequently. Each Organizational unit performs one or more Functions, and each Function can be done by more than one Organizational unit. An object called Responsible-for-OU identifies the person that coordinates all the activities that are performed at the Organizational unit. A person can be responsible for more than one Organizational Unit; this is expressed by the one or more constraint at the origin of the responsible link.

The use of this metamodel assumes a transitivity relationship. Let $\mathrm{A}$ and $\mathrm{B}$ be two Organizational unit objects, such that A is composed-of B. If B performs Function X, then X is also performed by A. However, the converse relationship is not always true because an Organizational unit component not necessarily performs all the Functions associated with the whole unit.

\subsection{Function-Task Metamodel}

Another basic metamodel, the so-called Function-Task metamodel presented in Figure 6, focuses on functions. Identification of functions should be independent of the current organization chart. An organization may change but still have to carry out the same functions. Metamodel functions can be further decomposed ("composed-of" link). For example, the Engineering function may be decomposed into Product Development and Process Development.

The objectives of a Function are achieved through the sequential and/or parallel execution of Tasks. A Task can be defined as an operation or a specific thing to be done. Tasks differ from Functions from a time-related point of view. Whereas functions are continuously performed, tasks have precise starting and finishing times. At the Task level, all the resources 
required and generated by a given task are explicitly represented. From this knowledge, all the resources associated to the accomplishment of a Function can be inferred.

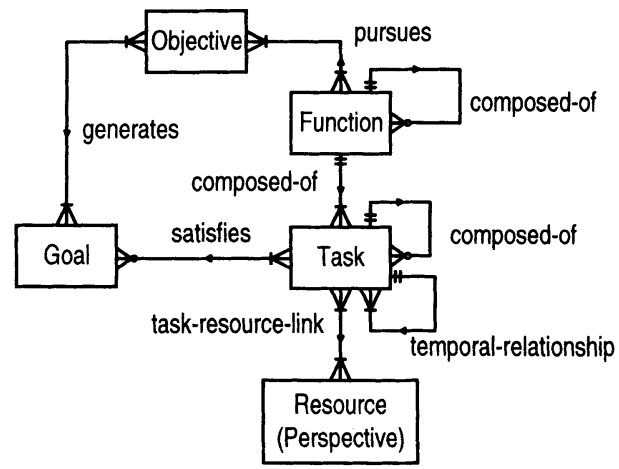

Figure 6 Function-Task metamodel.

A Task describes, in different abstraction levels, operations to be done. So, Tasks can be decomposed into subtasks, and these ones into new subtasks. The decomposition process is recursive; it finishes when the level of elementary tasks is reached (closure condition). The Task complexity degree determines the level of decomposition. In certain situations, there may be alternative ways in which a given Task can be solved. Therefore, a Task may have different decomposition structures. For example, a given scheduling task can be carried out differently if distinct goals are pursued. One decomposition structure is used if the reduction of scrap material goal is sought and another one if the reduction of work-in-process inventory is pursued.

According to the proposed metamodel, a Function is associated with a set of Objectives. An object called Objective identifies an aim or purpose of the organization. It can be pursued by more than one Function. The objectives associated with a Function are translated, at the level of tasks, into a set of Goals ("generates" relationship). Goals are specific targets that are intended to be reached by a given time. According to their time horizon they can be classified as strategic, tactic and operative.

Task decomposition structures provide an appropriate mechanism to represent the way in which tasks are solved. However, they are not sufficient to express coordination knowledge concerning the order in which subtasks are to be solved. Thus, establishing temporal links among tasks is very important. In this contribution, they are modeled by the temporal relationships proposed by Allen (1983). In order to represent them the temporal-relationship object was defined. It is specialized into the subclasses: before, equal, meets, overlaps, during, starts, and finishes. These links express precedence relationships among tasks as well as constraints that apply when resources are assigned to tasks.

Another main object in this metamodel is Resource that describes the elements that are created, consumed, used, produced, modified or eliminated by a Task during its execution in order to satisfy the task's Goals. As seen in Figure 7 the metaclass Resource (belonging to the Metamodel layer) has been instantiated into the following classes: Information (data items, files, drawings, messages, etc.), Material-Utility-Supply (products, steam, parts, electricity, 
etc.), Device (tools, machines, software packages, etc.) and General-resource (people, etc.), all of them belonging to the Model layer. Thus, in this layer, Resource makes reference to both information and physical resources. Resource instances can be further specialized. For example, Utility (Steam, Electricity, Compressed Air, Cooling Water, etc.) and Supply (Packaging Material, Lubricants, etc.) are subclasses of Material-Utility-Supply. Similarly, Device can be specialized into Equipment-item, Tool, Software Package, etc.

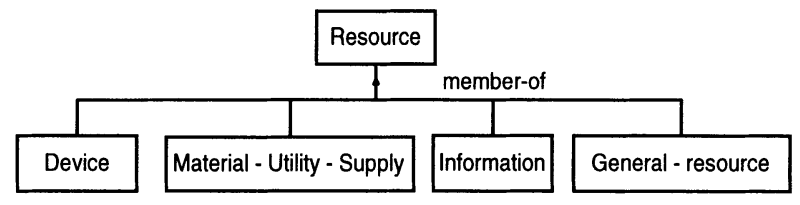

Figure 7 Some Resource instances in the Model Layer

The links that connect Tasks with Resources have been generalized by the task-resourcelink object, that is a specialization of the Relationship Class. This object expresses the fact that a task may be related to one or more resources, and that a given resource is associated with one or more tasks. The degree of abstraction adopted to model a Task determines the degree of complexity in which the associated resources are represented. For example, at a given level of abstraction it may be considered that a task named Manufacture-product-A uses the Production-line- $X$ resource. However, when this same task is modeled in greater detail the Production-line- $X$ resource can be expressed in terms of its constituent equipment items.

As it was mentioned before, a link that connects a Resource with a Task defines a specific resource role in relation to the task. The meaning of the different task-resource classes that specialize task-resource-link (See Figure 8) is given by the following definitions:

- uses. It represents the fact that a resource is utilized during the time interval in which the task is performed. During the task execution period the resource changes its state, but the initial and final states are the same. This link is specialized into the employs relation which indicates that the resource is exclusively used by one task. For example, if an equipment item is employed by a task, it would suffer the following changes of state: idle - busy - idle.

- consumes. It is applicable to consumable resources, indicating that a given amount of a resource disappears when the task is finished. Since the resource consumption implies its transformation into new resources, this relationship is pertinent to any resource that satisfies conservation laws.

- produces. Indicates the creation of a new resource. It is the converse relation of consumes. So, it is also applicable to resource classes that satisfy conservation laws.

- creates. The use of this link implies the creation of a new resource without satisfying any conservation law. For example, this link may represent the generation of a new version of an existing information resource, such as a new product design, as it was previously discussed.

- eliminates. It is creates converse relationship. This relation indicates that the resource disappears definitively (without satisfying any conservation law) when the task is finished.

- modifies. This relationship indicates that, as a result of the task execution, the resource suffers a change of state. 


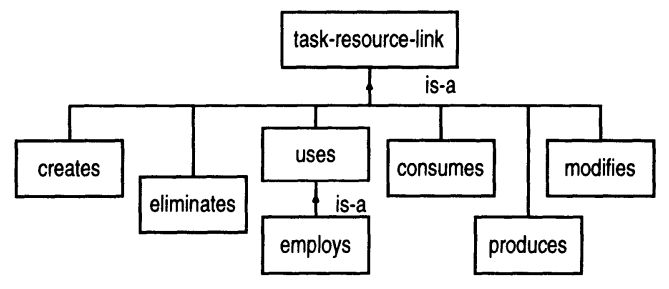

Figure 8 A taxonomy of the task-resource-link.

\section{DOMAIN APPLICATION MODELS}

The purpose of this section is to show the usage of the metamodel level primitives to represent the knowledge of specific production facilities. First, a series of generic models was constructed in the intermediate Model layer. These models can be made applicable to different types of organizations by properly specializing them.

In order to build these models specific questionnaires were generated. They were aimed at obtaining the knowledge necessary to describe the organization under analysis. Specifically, questionnaires try to identify: (i) Organizational units, (ii) Functions performed in the organization, (iii) Material and information flows linking the different functions, (iv) Information needs associated with the distinct hierarchical levels of the organization. In particular, for each function carried out in the production enterprise, questions such as the following were intended to be answered:

- main function objectives;

- lower level functions associated with it;

- tasks required to carry out each function;

- tasks' decomposition structures;

- tasks' precedence relationships;

- physical and information resources required by each task;

- physical and information resources generated by each task.

\subsection{Example}

Let us now consider the case of the XYZ production enterprise. Questionnaire answers revealed all the functions that are carried out by the six organizational units that comprise the $\mathrm{XYZ}$ company. One of functions was taken as an example because there is no available model for it in the Model layer. Then, it was necessary to instantiate a new one by using the Metamodel layer primitives. The chosen function is Production Planning and Control, that will be modeled as an instance of the Function-Task metamodel described in Figure 6.

A partial view of the resulting model is shown in Figure 9. The model provides a description of the function Production Planning and Control in terms of a hierarchy of functions: Production Control, Production Planning and Inventory Management. Production Planning consists of the subtasks (i) Create Aggregate Plan, (ii) Create Master Plan, (iii) Create 
Material Requirements Plan (MRP), and (iv) Create Schedule. In addition, it expresses the fact that the generation of a schedule depends on the realization of the task Create MRP, and that the latter task has to be started after the completion of the Create Master Plan task ("before ${ }_{2}$ " link). The same idea applies to the two other tasks related by the "before" link. Figure 10 presents an explosion of the Create MRP task introduced in the previous model. According to this figure, after subsequent decompositions Create MRP originates the tasks Determine Gross Requirements, and Determine Net Requirements. Moreover, to completely describe Create MRP, Figure 10 shows the resources that are needed to carry out the task (Master Plan, Product Structure, Product Lead Times, etc.) as well as the outputs generated by it (Raw Material Purchasing Agenda, Final and Intermediate Products Production Agenda, etc.).

Having developed these generic models, the actual models of the XYZ company can be generated and values to all the object attributes can be assigned. It should be mentioned that, for the sake of clarity, models shown in Figures 9 and 10 do not include cardinality constraints.

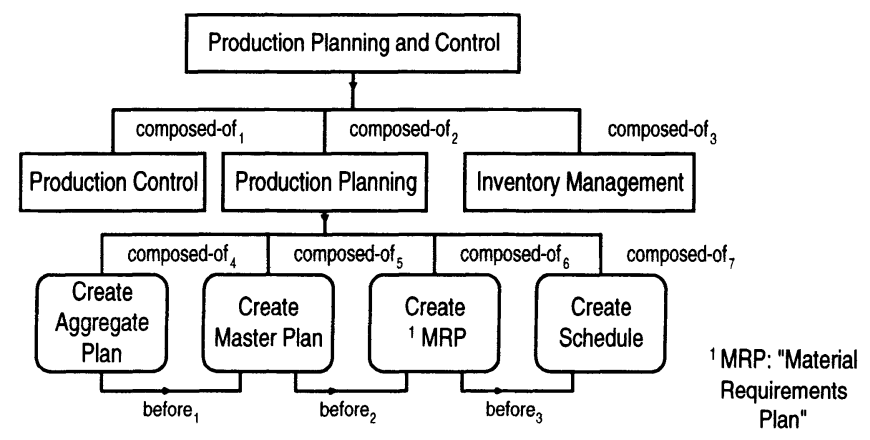

Figure 9 A partial view of the Function-Task metamodel which describes the Production Planning and Control function.

\section{CONCLUSIONS}

This paper presents advances in the development of an open model architecture consisting of three layers: (i) a Metamodel layer, where metamodels are defined, (ii) a Model layer associated with production enterprise generic models and (iii) a Universe of Discourse layer related to real world and conceptual entities that constitute the actual models of specific production facilities. The work focuses on the introduction of task and domain metamodels (based on the Object Technology), that are being used as templates in the construction of a model library. The proposed architecture is said to be open because (i) Modeling primitives within the metamodel layer can be specialized, (ii) New generic models, pertaining to the intermediate level, can be generated via metamodel instantiation, (iii) New generic models can be created by specializing existing generic models. The proposed architecture is being tested by modeling a small food company. 


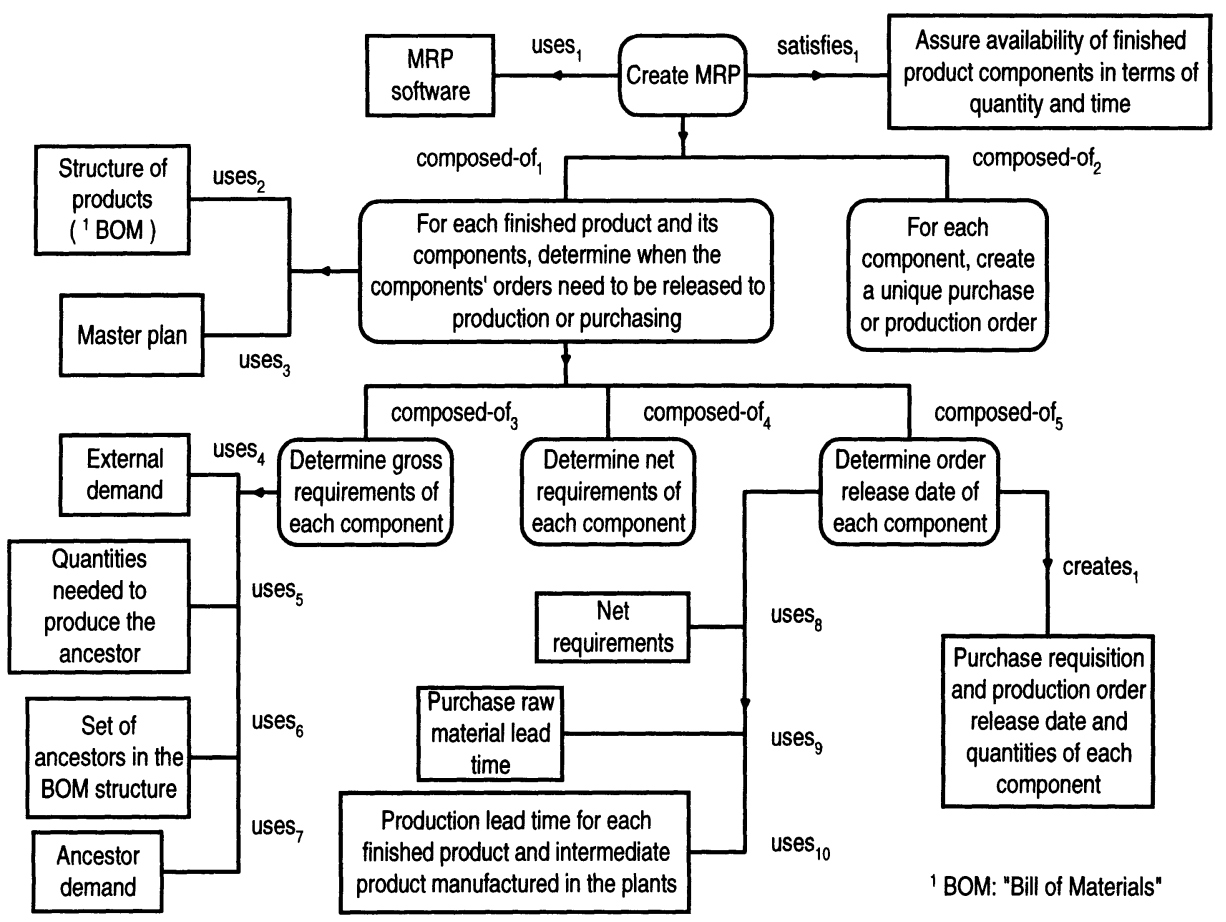

Figure 10 Explosion of the high level function shown in Figure 9.

\section{REFERENCES}

Allen J.F. (1983) Maintaining knowledge about temporal intervals. Communications of the $A C M, 26,832-843$.

Berio G., Di Leva A., Giolito P. and Vernadat F. (1995) The M*-OBJECT Methodology for Information System Design in CIM Environments. IEEE Trans. on Sys., Man and Cybern., 25, 68-85.

Booch G. (1994) Object-oriented analysis and design with applications. BenjaminCummings. New York

Jacobson I., Christerson M., Jonsson P. and Övergaad G. (1994) Object-Oriented Software Engineering. A Use Case Driven Approach. ACM Press. Addison-Wesley.

Kiczales G., des Rivières J. and Bobrow D.G. (1991) The Art of the Metaobject Protocol. The MIT Press.

Li Q. and Mc. Leod D. (1994) Conceptual Database Evolution through Learning in Object Databases. IEEE Trans. Knowledge and Data Eng., 6, 205-24.

Martin J. and Odell J. (1992) Object Oriented Analysis and Design. Prentice-Hall, Inc.

Mattsson S.E. (1988) On Model Structuring Concepts, in 4th IFAC Symposium on ComputerAided Design in Control Systems (CADCS), August 23-25. P. R. of China. 
Meyer W. (1990) Expert systems in Factory Management. Knowledge-Based CIM. Ellis Horwood Limited.

Petrie C. J. (Ed) (1992) Enterprise Integration Modeling. Proceedings of the First International Conference. The MIT Press.

Stefik M. and D. Bobrow (1985) Object-Oriented Programming: Themes and Variations. The AI Magazine, 6, 40-62.

Stephanopoulos G., Henning G. and Leone H. (1990) MODEL.LA. A Modeling Language for Process Engineering. Part I: The formal Framework. Part II: Multifaceted Modeling of Processing Systems. Comp. \& Chem. Engng, 14, 813-45, 847-69.

Vernadat F. (1988) Artificial Intelligence. Implications for Computer Integrated Manufacturing, in Artificial Intelligence in CIM Data Bases (ed. A. Kusiak). IFS Ltd. and Springer Verlag.

Vernadat F. B. (1992) CIMOSA - A European Development for Enterprise Integration. Part 2: Enterprise Modelling in Enterprise Integration Modeling. Proceedings of the First International Conference, The MIT Press, 189-204.

Ziegler B.P. (1984) Multifacetted Modelling and Discrete Event Simulation, Academic Press, London.

\section{BIOGRAPHIES}

Gabriela S. Mannarino is a Research Fellow of "Consejo Nacional de Investigaciones Científicas y Técnicas" (CONICET) at INGAR (CONICET - Fundación ARCIEN). She holds an Information Systems Engineering Degree from "Universidad Tecnológica Nacional, Fac. Reg. Santa Fe" (1993). She is currently working on object oriented analysis and design methodologies and the development of integrated information systems for production environments.

Gabriela P. Henning is an Assistant Professor and Researcher at INTEC (CONICET-Universidad Nacional del Litoral), Santa Fe, Argentina. She received a Chemical Engineering Degree from "Universidad Tecnológica Nacional, Fac. Reg. Rosario" (Argentina) in 1981 and earned her Ph.D. in Chemical Engineering from "Universidad Nacional del Litoral" in 1986. She was a Postdoctoral Fellow at the Massachusetts Institute of Technology (1986-1989), specializing in Applications of Artificial Intelligence in Process Engineering. Her research interests include the application of knowledge-based and mathematical programming methodologies to industrial scheduling problems, the development of high-level languages for process engineering activities and the design of integrated information systems for production environments.

Horacio P. Leone holds possitions as Associate Professor at the Information Systems Department, Universidad Tecnológica Nacional Fac. Reg. Santa Fe, and Researcher at INGAR (CONICETFundación ARCIEN), Santa Fe, Argentina. He received a Chemical Engineering Degree from "Universidad Tecnológica Nacional, Fac. Reg. Rosario" (Argentina) in 1981, and earned his Ph.D. in Chemical Engineering from "Universidad Nacional del Litoral" in 1986. He was a Postdoctoral Fellow at the Massachusetts Institute of Technology (1986-1989), specializing in Applications of Artificial Intelligence in Process Engineering. His current research focuses on object-oriented analysis and design of information systems for process industries and the application of artificial intelligence methodologies in decision support systems for fault diagnosis. 